IJMMS 26:10 (2001) 625-634

PII. S0161171201004628

http://ijmms.hindawi.com

(c) Hindawi Publishing Corp.

\title{
ON A NONRESONANCE CONDITION BETWEEN THE FIRST AND THE SECOND EIGENVALUES FOR THE $p$-LAPLACIAN
}

\author{
A. ANANE and N. TSOULI
}

(Received 24 February 2000)

\begin{abstract}
We are concerned with the existence of solution for the Dirichlet problem $-\triangle_{p} u=f(x, u)+h(x)$ in $\Omega, u=0$ on $\partial \Omega$, when $f(x, u)$ lies in some sense between the first and the second eigenvalues of the $p$-Laplacian $\triangle_{p}$. Extensions to more general operators which are $(p-1)$-homogeneous at infinity are also considered.
\end{abstract}

2000 Mathematics Subject Classification. 35J65.

1. Introduction. In this paper, we are concerned with the existence of solution to the following quasilinear elliptic problem:

$$
\begin{gathered}
-\triangle_{p} u=f(x, u)+h(x) \text { in } \Omega, \\
u=0 \quad \text { on } \partial \Omega .
\end{gathered}
$$

Here $\Omega$ is a smooth bounded domain of $\mathbb{R}^{N}, N \geq 1, \Delta_{p}$ denotes the $p$-Laplacian $\Delta_{p} u=$ $\operatorname{div}\left(|\nabla u|^{p-2} \nabla u\right), 1<p<\infty, h$ belongs to $W^{-1, p^{\prime}}(\Omega)$ with $p^{\prime}$ the Hölder conjugate of $p$ and $f$ is a Caratheodory function from $\Omega \times \mathbb{R}$ to $\mathbb{R}$ such that

$$
\lambda_{1} \underset{\equiv}{\leq} \liminf _{s \rightarrow \pm \infty} \frac{f(x, s)}{|s|^{p-2} s} \leq \limsup _{s \rightarrow \pm \infty} \frac{f(x, s)}{|s|^{p-2} s}<\lambda_{2} \quad \text { a.e. in } \Omega,
$$

where $\lambda_{1}$ (resp., $\lambda_{2}$ ) is the first (resp., the second) eigenvalue of the problem

$$
\begin{gathered}
-\Delta_{p} u=\lambda|u|^{p-2} u \quad \text { in } \Omega, \\
u=0 \quad \text { on } \partial \Omega .
\end{gathered}
$$

Problems of this sort have been extensively studied in the 70s and 80s in the semilinear case $p=2$. In the quasilinear case $p \neq 2$, (1.1) was investigated for $N=1$ in [6] and for $N \geq 1$ in [3]. In this latter work nonresonance is studied at the left of $\lambda_{1}$.

One of the difficulties to deal with the partial differential equation case $N \geq 1$ is the lack of knowledge of the spectrum of the $p$-Laplacian in that case. The basic properties of $\lambda_{1}$ were established in [2], while a variational characterization of $\lambda_{2}$ was derived recently in [4]. This variational characterization of $\lambda_{2}$ allows the study of its (strict) monotonicity dependence with respect to a weight. This is the property which is used in our approach to (1.1). The asymmetry in our assumption (1.2) between $\lambda_{1}$ and $\lambda_{2}$ also comes from that property. In fact it remains an open question whether the last strict inequality in (1.2) can be replaced by $\underset{\equiv}{\leq}$. 
In Section 3 we extend our existence result to more general operators. We consider

$$
\begin{gathered}
A(u)=f(x, u)+h(x) \text { in } \Omega, \\
u=0 \quad \text { on } \partial \Omega,
\end{gathered}
$$

where $A=-\sum_{i=1}^{N}\left(\partial / \partial x_{i}\right) A_{i}(x, u, \nabla u)$ verifies a $(p-1)$-homogeneity condition at infinity. Such operators were studied by Anane [1] in the variational case. Here we use degree theory for mappings of type $(S)_{+}$as developed by Browder [7] and Berkowits and Mustonen [5]. No variational structure is consequently needed.

2. A result for the $p$-Laplacian. We seek a weak solution of (1.1), that is,

$$
\begin{aligned}
& \text { find } u \in W_{0}^{1, p}(\Omega) \quad \text { such that } \quad \forall v \in W_{0}^{1, p}(\Omega): \\
& \int_{\Omega}|\nabla u|^{p-2} \nabla u . \nabla v d x=\int_{\Omega} f(x, u) v d x+\langle h, v\rangle,
\end{aligned}
$$

where $\langle\cdot, \cdot\rangle$ denotes the duality product between $W^{-1, p^{\prime}}(\Omega)$ and $W_{0}^{1, p}(\Omega)$. We assume that $f$ satisfies

$$
\begin{gathered}
\max _{|\mathcal{S}| \leq R}|f(x, s)| \in L^{p^{\prime}}(\Omega), \quad \forall R>0, \\
\lambda_{1} \leq l(x) \leq k(x)<\lambda_{2} \quad \text { a.e. in } \Omega,
\end{gathered}
$$

where

$$
l(x)=\liminf _{s \rightarrow \pm \infty} \frac{f(x, s)}{|s|^{p-2} s}, \quad k(x)=\limsup _{s \rightarrow \pm \infty} \frac{f(x, s)}{|s|^{p-2} s} .
$$

The first inequality in (2.3) must be understood as "less or equal almost everywhere together with strict inequality on a set of positive measure." We also assume that some uniformity holds in the inequalities in (2.3):

$$
\begin{aligned}
& \forall \varepsilon>0, \quad \exists \eta(\varepsilon)>0: \lambda_{1}-\varepsilon \leq \frac{f(x, s)}{|s|^{p-2} s}, \quad \forall|s| \geq \eta(\varepsilon), \quad \text { a.e. in } \Omega, \\
& \forall \varepsilon>0, \quad \exists \eta(\varepsilon)>0: \frac{f(x, s)}{|s|^{p-2} s} \leq \lambda_{2}+\varepsilon, \quad \forall|s| \geq \eta(\varepsilon), \quad \text { a.e. in } \Omega \text {. }
\end{aligned}
$$

REMARK 2.1. It is clear that (2.2) and (2.5) imply the growth condition

$$
|f(x, s)| \leq a|s|^{p-1}+b(x) \quad \forall s \in \mathbb{R} \text {, a.e. in } \Omega,
$$

where $a>0$ and $b(\cdot) \in L^{p^{\prime}}(\Omega)$.

REMARK 2.2. Equations (2.2) and (2.5) also imply

$$
\begin{aligned}
& \forall \varepsilon>0, \quad \exists b_{\varepsilon} \in L^{p^{\prime}}(\Omega) \text { such that } \\
& \qquad \begin{array}{c}
|s|^{p}\left(\lambda_{1}-\varepsilon\right)-b_{\varepsilon}(x) \leq s f(x, s) \leq|s|^{p}\left(\lambda_{2}+\varepsilon\right)+b_{\varepsilon}(x), \\
\forall s \in \mathbb{R}, \quad \text { a.e. in } \Omega .
\end{array}
\end{aligned}
$$


TheOrem 2.3. Suppose that $f$ satisfies (2.2), (2.3), and (2.5). Then for any $h \in$ $W^{-1, p^{\prime}}(\Omega)$, problem (2.1) admits a solution $u$ in $W_{0}^{1, p}(\Omega)$.

Proof. We denote by $\left(T_{t}\right)_{t \in[0,1]}$ the family of operators from $W_{0}^{1, p}(\Omega)$ to $W_{0}^{1, p}(\Omega)$ defined by

$$
T_{t}(u)=\left(-\Delta_{p}\right)^{-1}\left[(1-t) \alpha|u|^{p-2} u+t f(\cdot, u)+t h(\cdot)\right],
$$

where $\alpha$ is some fixed number with $\lambda_{1}<\alpha<\lambda_{2}$.

To prove Theorem 2.3, we first establish the following estimate:

$$
\exists R>0 \text { such that } \forall t \in[0,1], \forall u \in \partial B(O, R) \text { such that }\left[I-T_{t}\right](u) \neq 0 \text {, }
$$

where $B(O, R)$ denotes the ball of center $O$ and radius $R$ in $W_{0}^{1, p}(\Omega)$.

To prove (2.9) we assume by contradiction that

$$
\forall n>0, \quad \exists t_{n} \in[0,1], \exists u_{n} \in W_{0}^{1, p}(\Omega) \text { with }\left\|u_{n}\right\|_{1, p}=n \text { such that } T_{t_{n}}\left(u_{n}\right)=u_{n},
$$

where $\|\cdot\|_{1, p}$ denotes the norm in $W_{0}^{1, p}(\Omega)$.

Let $w_{n}=u_{n} / n$. We can extract from $\left(w_{n}\right)$ a subsequence, still denoted by $\left(w_{n}\right)$, which converges weakly in $W_{0}^{1, p}(\Omega)$, strongly in $L^{p}(\Omega)$ and a.e. in $\Omega$ to $w \in W_{0}^{1, p}(\Omega)$. We can also suppose that $t_{n}$ converges to $t \in[0,1]$. To reach a contradiction, we use the following lemmas which give various information on $w_{n}$ and $w$.

LEMMA 2.4. The sequence $g_{n}$ defined by

$$
g_{n}=\frac{f\left(x, n w_{n}\right)}{n^{p-1}}
$$

is bounded in $L^{p^{\prime}}(\Omega)$, and consequently, for a subsequence, $g_{n}$ converges weakly to some $g$ in $L^{p^{\prime}}(\Omega)$.

Proof. This is an immediate consequence of (2.6).

LEMMA 2.5. $w \neq \equiv$.

Proof. Since $w_{n}$ verifies,

$$
\begin{aligned}
\int_{\Omega}\left|\nabla w_{n}\right|^{p} d x= & \left(1-t_{n}\right) \alpha \int_{\Omega}\left|w_{n}\right|^{p} d x \\
& +t_{n}\left[\int_{\Omega} g_{n}(x) w_{n}(x) d x+\frac{1}{n^{p-1}}\left\langle h, w_{n}\right\rangle\right],
\end{aligned}
$$

we deduce from Lemma 2.4 that

$$
1=(1-t) \alpha \int_{\Omega}|w|^{p} d x+t \int_{\Omega} g(x) w(x) d x,
$$

which clearly implies the conclusion of Lemma 2.5.

LEMMA 2.6. $g=0$ a.e. in $\Omega \backslash A$, where $A=\{x \in \Omega: w(x) \neq 0\}$. 
Proof. By (2.6), we have

$$
\left|g_{n}(x)\right| \leq a\left|w_{n}\right|^{p-1}+\frac{b(x)}{n^{p-1}} \quad \text { a.e. in } \Omega,
$$

and so

$$
\left\|g_{n}\right\|_{L^{p^{\prime}}(\Omega \backslash A)} \leq a\left\|w_{n}\right\|_{L^{p}(\Omega \backslash A)}^{p / p^{\prime}}+\frac{1}{n^{p-1}}\|b\|_{L^{p^{\prime}}(\Omega \backslash A)},
$$

which implies

$$
\lim _{n \rightarrow+\infty}\left\|g_{n}\right\|_{L^{p^{\prime}}(\Omega \backslash A)}=0 .
$$

Set $D=\{x \in \Omega \backslash A: g(x) \neq 0\}$. By Lemma 2.4 we have, for $\phi(x)=\operatorname{sign}[g(x)] \chi_{D}(x) \in$ $L^{p}(D)$

$$
\lim _{n \rightarrow+\infty} \int_{D} g_{n}(x) \phi(x) d x=\int_{D}|g(x)| d x,
$$

and consequently by (2.16),

$$
\int_{D}|g(x)| d x=0
$$

which implies meas $(D)=0$, that is, the conclusion of Lemma 2.6.

LEMMA 2.7. Set

$$
\tilde{g}(x)= \begin{cases}\frac{g(x)}{|w(x)|^{p-2} w(x)} & \text { on } A, \\ \beta & \text { on } \Omega \backslash A,\end{cases}
$$

where $\beta$ is a fixed number with $\lambda_{1}<\beta<\lambda_{2}$. We have

$$
\lambda_{1} \underset{\ddagger}{\mathfrak{g}}(x)<\lambda_{2} \quad \text { a.e. in } \Omega \text {. }
$$

Proof. Set

$$
\begin{aligned}
& B_{l}=\left\{x \in A: w(x) g(x)<l(x)|w(x)|^{p}\right\}, \\
& B_{k}=\left\{x \in A: w(x) g(x)>k(x)|w(x)|^{p}\right\} .
\end{aligned}
$$

We first prove that meas $\left(B_{l}\right)=0$ and meas $\left(B_{k}\right)=0$.

By (2.7), we have that $\forall \varepsilon \geq 0, \exists b_{\varepsilon} \in L^{p^{\prime}}(\Omega)$ such that

$$
\begin{aligned}
-\frac{b_{\varepsilon}(x)}{n^{p}} & +\left|w_{n}(x)\right|^{p}[l(x)-\varepsilon] \\
& \leq w_{n}(x) g_{n}(x) \leq \frac{b_{\varepsilon}(x)}{n^{p}}+\left|w_{n}(x)\right|^{p}[k(x)+\varepsilon] \text { a.e. in } \Omega .
\end{aligned}
$$

The first inequality gives

$$
-\frac{1}{n^{p}} \int_{B_{l}} b_{\varepsilon}(x) d x+\int_{B_{l}}\left|w_{n}(x)\right|^{p}[l(x)-\varepsilon] d x \leq \int_{B_{l}} w_{n}(x) g_{n}(x) d x .
$$

Letting first $x \rightarrow \infty$, then $\varepsilon \rightarrow 0$, we deduce

$$
\int_{B_{l}}\left[w(x) g(x)-|w(x)|^{p} l(x)\right] d x \geq 0
$$


which implies meas $\left(B_{l}\right)=0$. Similarly one gets meas $\left(B_{k}\right)=0$. We thus have

$$
l(x) \leq \tilde{g}(x) \leq k(x) \text { a.e. in } A .
$$

Since

$$
\lambda_{1}<\tilde{g}(x)=\beta<\lambda_{2} \quad \text { a.e. in } \Omega \backslash A,
$$

we obtain the conclusion of the lemma.

LEMMA 2.8. $w$ is a solution of

$$
\begin{gathered}
-\Delta_{p} w=m|w|^{p-2} w \quad \text { in } \Omega, \\
w=0 \quad \text { on } \partial \Omega,
\end{gathered}
$$

where $m(x)=(1-t) \alpha+t \tilde{g}(x)$.

PROof. We first prove that $w$ is a solution of

$$
\begin{gathered}
-\Delta_{p} w=(1-t) \alpha|w|^{p-2} w+t g \text { in } \Omega, \\
w=0 \quad \text { on } \partial \Omega .
\end{gathered}
$$

We recall that $w_{n}$ satisfies

$$
\begin{gathered}
-\Delta_{p} w_{n}=\left(1-t_{n}\right) \alpha\left|w_{n}\right|^{p-2} w_{n}+t_{n}\left[g_{n}+\frac{1}{n^{p-1}} h\right] \quad \text { in } \Omega, \\
w_{n}=0 \quad \text { on } \partial \Omega .
\end{gathered}
$$

Since $\left(-\Delta_{p}\right)\left(w_{n}\right)$ is bounded in $W^{-1, p^{\prime}}(\Omega)$, there exists a subsequence, still denoted by $\left(w_{n}\right)$, and a distribution $T \in W^{-1, p^{\prime}}(\Omega)$, such that $\left(-\Delta_{p}\right)\left(w_{n}\right)$ converges weakly to $T$ in $W^{-1, p^{\prime}}(\Omega)$; in particular

$$
\lim _{n \rightarrow+\infty}\left\langle-\Delta_{p} w_{n}, w\right\rangle=\langle T, w\rangle
$$

We also have

$$
\begin{aligned}
\left\langle-\Delta_{p} w_{n}, w_{n}-w\right\rangle= & \left(1-t_{n}\right) \alpha \int_{\Omega}\left|w_{n}\right|^{p-2} w_{n}\left(w_{n}-w\right) d x \\
& +t_{n}\left[\int_{\Omega} g_{n}(x)\left(w_{n}-w\right) d x+\frac{1}{n^{p-1}}\left\langle h, w_{n}-w\right\rangle\right],
\end{aligned}
$$

which implies

$$
\lim _{n \rightarrow+\infty}\left\langle-\Delta_{p} w_{n}, w_{n}-w\right\rangle=0
$$

and therefore

$$
\lim _{n \rightarrow+\infty}\left\langle-\Delta_{p} w_{n}, w_{n}\right\rangle=\langle T, w\rangle .
$$

Since $\left(-\Delta_{p}\right)$ is an operator of type $(M)$, we deduce

$$
T=-\Delta_{p} w
$$


Going to the limit in (2.29) then yields (2.28). But by Lemma 2.6, we have

$$
(1-t) \alpha|w|^{p-2} w+t g=m|w|^{p-2} w \quad \text { a.e. in } \Omega .
$$

So $w$ is a solution of (2.27).

We denote by $\lambda_{1}(\Omega, r(x))$ (resp., $\left.\lambda_{2}(\Omega, r(x))\right)$ the first (resp., the second) eigenvalue in the problem with weight

$$
\begin{gathered}
-\Delta_{p} u=\lambda r(x)|u|^{p-2} u \quad \text { in } \Omega, \\
u=0 \quad \text { on } \partial \Omega .
\end{gathered}
$$

By Lemma 2.7 and the fact that $\lambda_{1}<\alpha<\lambda_{2}$, we have

$$
\lambda_{1} \underset{\equiv}{\lessgtr} m(x)<\lambda_{2} \quad \text { a.e. in } \Omega \text {. }
$$

It follows, by the strict monotonicity property of the second eigenvalue with respect to the weight (cf. [4]), that

$$
1=\lambda_{2}\left(\Omega, \lambda_{2}\right)<\lambda_{2}(\Omega, m)
$$

It also follows by the strict monotonicity of the first eigenvalue with respect to the weight (cf. [8]), that

$$
\lambda_{1}(\Omega, m)<\lambda_{1}\left(\Omega, \lambda_{1}\right)=1 .
$$

Consequently,

$$
\lambda_{1}(\Omega, m)<1<\lambda_{2}(\Omega, m) .
$$

But by Lemmas 2.5 and 2.8, 1 is an eigenvalue of $\left(-\Delta_{p}\right)$ for the weight $m$. This contradicts the definition of the second eigenvalue $\lambda_{2}(\Omega, m)$. We have thus proved that the estimate (2.9) holds.

We can now conclude by a standard degree argument. Indeed $T_{t}$ is clearly completely continuous, since $\left(\Delta_{p}\right)^{-1}$ is continuous from $W^{-1, p^{\prime}}(\Omega)$ to $W_{0}^{1, p}(\Omega)$. Therefore,

$$
\operatorname{deg}\left(I-T_{0}, B(O, R), O\right)=\operatorname{deg}\left(I-T_{1}, B(O, R), O\right) .
$$

Since $T_{0}$ is odd, we have, by Borsuk theorem, that $\operatorname{deg}\left(I-T_{0}, B(O, R), O\right)$ is an odd integer and so nonzero. It then follows that there exists $u \in B(O, R)$ such that $T_{1}(u)=u$, which proves Theorem 2.3.

3. Generalization. Theorem 2.3 will now be extended to the case of nonhomogeneous operators. We consider the problem

$$
\begin{gathered}
A(u)=f(x, u)+h(x) \text { in } \Omega, \\
u=0 \text { on } \partial \Omega,
\end{gathered}
$$

where

$$
A(u)=-\sum_{i=1}^{N} \frac{\partial}{\partial x_{i}} A_{i}(x, u(x), \nabla u(x))
$$


The method used in Section 2 for $\left(-\Delta_{p}\right)$ can be adapted under suitable assumptions on $A$. We basically assume that $A$ is a Leray-Lions operator which is $(p-1)$ homogeneous at infinity. Our precise assumptions are the following:

Each $A_{i}(x, s, \xi)$ is a Carathéodory function,

$\sum_{i=1}^{N}\left[A_{i}(x, s, \xi)-A_{i}\left(x, s, \xi^{\prime}\right)\right]\left(\xi_{i}-\xi_{i}^{\prime}\right)>0$, for a.e. $x \in \Omega$, all $s \in \mathbb{R}$, all $\xi \neq \xi^{\prime} \in \mathbb{R}^{N}$,

$\exists K \in L^{p^{\prime}}(\Omega), \exists c(t)$ a function defined on $\mathbb{R}^{+}$with $\lim _{t \rightarrow+\infty} c(t)=0$ such that

$$
\left.\left|A_{i}(x, t s, t \xi)-t^{p-1}\right| \xi\right|^{p-2} \xi_{i} \mid \leq t^{p-1} c(t)\left[|\xi|^{p-1}+|s|^{p-1}+K(x)\right],
$$

for a.e. $x \in \Omega$, all $s \in \mathbb{R}$, all $\xi \in \mathbb{R}^{N}$, all $t \in \mathbb{R}^{+}$.

We will be able to solve (3.1) when $f(x, s)$ lies at infinity between the first and the second eigenvalues of the $p$-Laplacian $\left(-\Delta_{p}\right)$, in the sense of (1.2).

REMARK 3.1. Equation (3.5) is a hypothesis which means that $A$ is asymptotically homogeneous to $\left(-\Delta_{p}\right)$. An example of an operator which verifies (3.3), (3.4), and (3.5) is the following regularized version of the $p$-Laplacian:

$$
A=-\Delta_{p, \epsilon}=-\operatorname{div}\left[\left(\epsilon+|\nabla u|^{2}\right)^{(p-2) / 2} \nabla u\right]
$$

with $\epsilon>0$.

REMARK 3.2. Equations (3.3), (3.4), and (3.5) imply the following usual growth and coercivity conditions:

$$
\begin{gathered}
\exists c_{4}>0, \exists K_{4} \in L^{p^{\prime}}(\Omega) \text { such that }\left|A_{i}(x, s, \xi)\right| \leq c_{4}\left(|\xi|^{p-1}+|s|^{p-1}+K_{4}(x)\right), \\
\text { a.e. } x \in \Omega, \forall s \in \mathbb{R}, \xi \in \mathbb{R}^{N} \text {, for } i=1, \ldots, N, \\
\exists c_{5}>0, c_{5}^{\prime}>0, K_{5} \in L^{1}(\Omega) \text { such that } \sum_{i=1}^{N} A_{i}(x, s, \xi) \xi_{i} \geq c_{5}|\xi|^{p}-c_{5}^{\prime}|s|^{p}-K_{5}(x), \\
\text { a.e. } x \in \Omega, \forall s \in \mathbb{R}, \xi \in \mathbb{R}^{N} .
\end{gathered}
$$

Indeed (3.7) follows immediately from (3.5). To verify (3.8), one observes that by (3.5) one has, for each $t>0$,

$$
A_{i}(x, t s, t \xi) \xi_{i}-t^{p-1}|\xi|^{p-2} \xi_{i}^{2} \geq-t^{p-1} c(t)\left|\xi_{i}\right|\left[|\xi|^{p-1}+|s|^{p-1}+K(x)\right]
$$

and so

$$
\sum_{i=1}^{N} A_{i}(x, t s, t \xi) \xi_{i} \geq t^{p-1}|\xi|^{p}\left[1-N c(t)\left(1+\frac{2}{p}\right)\right]-\frac{1}{p^{\prime}} t^{p-1}|c(t)| N\left(|s|^{p}+|K(x)|^{p^{\prime}}\right) .
$$

Choosing $t$ sufficiently large yields (3.8). 
REMARK 3.3. Equations (3.3) and (3.5) imply that $A$ is well defined, continuous, and bounded from $W_{0}^{1, p}(\Omega)$ to $W^{-1, p^{\prime}}(\Omega)$. Equations (3.3), (3.4), and (3.5) also imply that $A$ is of type $(S)_{+}$. This latter fact can be proved along similar lines as in the argument given by Berkovits and Mustonen in [5].

We are now ready to state the following theorem.

Theorem 3.4. Assume (2.2), (2.3), (2.5), (3.3), (3.4), and (3.5). Then for any $h \in$ $W^{-1, p^{\prime}}(\Omega)$, there exists a weak solution $u \in W_{0}^{1, p}(\Omega)$ of (3.1), that is,

$$
\int_{\Omega} \sum_{i=1}^{N} A_{i}(x, u(x), \nabla u(x)) \frac{\partial v}{\partial x_{i}} d x=\int_{\Omega} f(x, u) v d x+\langle h, v\rangle, \quad \forall v \in W_{0}^{1, p}(\Omega) .
$$

Proof. The proof is rather similar to that of Theorem 2.3, and we will only detail below those points which really involve the operator $A$.

Let $\left(S_{t}\right)_{t \in[0,1]}$ be the family of operators from $W_{0}^{1, p}(\Omega)$ to $W^{-1, p^{\prime}}(\Omega)$ defined by

$$
S_{t}(u)=t A(u)-(1-t)\left(\Delta_{p} u\right)-t[f(x, u)+h(x)]-(1-t) \alpha|u|^{p-2} u,
$$

for some fixed number $\alpha$ with $\lambda_{1}<\alpha<\lambda_{2}$. Since the operator $A$ is of type $(S)_{+}, S_{t}$ is also of type $(S)_{+}$. By the degree theory for mappings of type $(S)_{+}$, as developed in Browder [7] and Berkowits and Mustonen [5], to solve (3.1) it suffices to prove the following estimate:

$$
\exists R>0 \text { such that } \forall t \in[0,1], \quad \forall u \in \partial B(O R) \text { such that } S_{t}(u) \neq 0 .
$$

To prove (3.13), we assume by contradiction that

$$
\forall n \in \mathbb{N}, \exists t_{n} \in[0,1], \exists u_{n} \in W_{0}^{1, p}(\Omega) \text { with }\left\|u_{n}\right\|_{1, p}=n \text {, such that } S_{t_{n}}\left(u_{n}\right)=0 \text {. }
$$

Let $w_{n}=u_{n} / n$. We can extract from $\left(w_{n}\right)$ a subsequence, still denoted by $\left(w_{n}\right)$, which converges weakly in $W_{0}^{1, p}(\Omega)$, strongly in $L^{p}(\Omega)$ and a.e. in $\Omega$ to $w \in W_{0}^{1, p}(\Omega)$. We can also suppose that $t_{n}$ converges to $t \in[0,1]$.

In the same manner as in the proof of Theorem 2.3, to obtain a contradiction, we use Lemmas 2.4, 2.6, and 2.7 (which do not involve the operator $A$ ) together with the following two lemmas.

LEMMA 3.5. $w \neq 0$.

Proof. By (3.14) we have

$$
\begin{aligned}
\left\langle\frac{t_{n} A\left(u_{n}\right)}{n^{p-1}}-\left(1-t_{n}\right) \Delta_{p} w_{n}, w_{n}\right\rangle= & \left(1-t_{n}\right) \alpha \int_{\Omega}\left|w_{n}\right|^{p} d x \\
& +t_{n}\left[\int_{\Omega} g_{n}(x) w_{n}(x) d x+\frac{1}{n^{p-1}}\left\langle h, w_{n}\right\rangle\right] .
\end{aligned}
$$


Since

$$
\begin{aligned}
& \left|\left\langle\frac{t_{n} A\left(u_{n}\right)}{n^{p-1}}-t_{n}\left(-\Delta_{p} w_{n}\right), w_{n}\right\rangle\right| \\
& \quad \leq\left. n^{1-p} \int_{\Omega} \sum_{i=1}^{N}\left|A_{i}\left(x, u_{n}, n \nabla w_{n}\right)-n^{p-1}\right| \nabla w_{n}\right|^{p-2} \frac{\partial w_{n}}{\partial x_{i}}|\cdot| \frac{\partial w_{n}}{\partial x_{i}} \mid d x,
\end{aligned}
$$

using (3.5) and the fact that $\left\|w_{n}\right\|_{1, p}=1$, we obtain

$$
\begin{aligned}
& \left|\left\langle\frac{t_{n} A\left(u_{n}\right)}{n^{p-1}}-t_{n}\left(-\Delta_{p} w_{n}\right), w_{n}\right\rangle\right| \\
& \quad \leq c(n)\left[\left\|\nabla w_{n}\right\|_{L^{p}(\Omega)}^{p / p^{\prime}}+\left\|w_{n}\right\|_{L^{p}(\Omega)}^{p / p^{\prime}}+\|K\|_{L^{p^{\prime}}(\Omega)}\right]\left\|w_{n}\right\|_{1, p} \stackrel{n \rightarrow+\infty}{\longrightarrow} 0 .
\end{aligned}
$$

Therefore

$$
1=(1-t) \alpha \int_{\Omega}|w|^{p} d x+t \int_{\Omega} g(x) w(x) d x,
$$

which clearly implies $w \neq 0$.

LEMMA 3.6. $w$ is a solution of

$$
\begin{gathered}
-\Delta_{p} w=m|w|^{p-2} w \quad \text { in } \Omega, \\
w=0 \quad \text { on } \partial \Omega,
\end{gathered}
$$

where $m(x)=((1-t) \alpha+t \tilde{g}(x))$ and $\tilde{g}$ is defined in Lemma 2.7.

Proof. We first show that $w$ is a solution of

$$
\begin{gathered}
-\Delta_{p} w=(1-t) \alpha|w|^{p-2} w+t g \text { in } \Omega, \\
w=0 \quad \text { on } \partial \Omega .
\end{gathered}
$$

Since $\left(-\Delta_{p}\right)\left(w_{n}\right)$ is bounded in $W^{-1, p^{\prime}}(\Omega)$, there exists a subsequence, still denoted by $\left(w_{n}\right)$, and a distribution $T \in W^{-1, p^{\prime}}(\Omega)$, such that $\left(-\Delta_{p}\right)\left(w_{n}\right)$ converges weakly to $T$ in $W^{-1, p^{\prime}}(\Omega)$. In particular

$$
\lim _{n \rightarrow+\infty}\left\langle-\Delta_{p} w_{n}, w\right\rangle=\langle T, w\rangle
$$

We also have

$$
\begin{aligned}
\left\langle-\Delta_{p} w_{n}, w_{n}-w\right\rangle= & \left(1-t_{n}\right) \alpha \int_{\Omega}\left|w_{n}\right|^{p-2} w_{n}\left(w_{n}-w\right) d x \\
& +t_{n}\left[\int_{\Omega} g_{n}(x)\left(w_{n}-w\right) d x+\frac{1}{n^{p-1}}\left\langle h, w_{n}-w\right\rangle\right] \\
& -\left\langle t_{n}\left[\frac{A\left(u_{n}\right)}{n^{p-1}}+\Delta_{p} w_{n}\right], w_{n}-w\right\rangle
\end{aligned}
$$


and since, by (3.5),

$$
\begin{aligned}
& \left|\left\langle t_{n}\left[\frac{A\left(u_{n}\right)}{n^{p-1}}+\Delta_{p} w_{n}\right], w_{n}-w\right\rangle\right| \\
& \quad \leq c(n)\left[\left\|\nabla w_{n}\right\|_{L^{p}(\Omega)}^{p / p^{\prime}}+\left\|w_{n}\right\|_{L^{p}(\Omega)}^{p / p^{\prime}}+\|K\|_{L^{p^{\prime}(\Omega)}}\right]\left\|w_{n}-w\right\|_{1, p} \stackrel{n \rightarrow+\infty}{\longrightarrow} 0,
\end{aligned}
$$

we deduce

$$
\lim _{n \rightarrow+\infty}\left\langle-\Delta_{p} w_{n}, w_{n}-w\right\rangle=0 .
$$

The rest of the proof of Lemma 3.6 uses the fact that $\left(-\Delta_{p}\right)$ is of type $(M)$ and is similar to the proof of Lemma 2.8.

ACKNOWLEDGEMENT. We wish to thank J.-P. Gossez for various interesting discussions on the subject of this paper.

\section{REFERENCES}

[1] A. Anane, Etude des valeurs propres et de la résonance pour l'opérateur p-Laplacien, Ph.D. thesis, Université Libre de Bruxelles, 1987.

[2] __ Simplicité et isolation de la première valeur propre du $p$-Laplacien avec poids [Simplicity and isolation of the first eigenvalue of the $p$-Laplacian with weight], C. R. Acad. Sci. Paris Sér. I Math. 305 (1987), no. 16, 725-728 (French). MR 89e:35124. Zbl 633.35061.

[3] A. Anane and J.-P. Gossez, Strongly nonlinear elliptic problems near resonance: a variational approach, Comm. Partial Differential Equations 15 (1990), no. 8, 1141-1159. MR 91h:35121. Zbl 715.35029.

[4] A. Anane and N. Tsouli, On the second eigenvalue of the p-Laplacian, Nonlinear Partial Differential Equations (Fès, 1994), Pitman Research Notes in Mathematics Series, vol. 343, Longman, Harlow, 1996, pp. 1-9. MR 97k:35190. Zbl 854.35081.

[5] J. Berkovits and V. Mustonen, Nonlinear Mappings of Monotone Type Classification and Degree Theory, University of Oulu, Finland, 1988.

[6] L. Boccardo, P. Drábek, D. Giachetti, and M. Kučera, Generalization of Fredholm alternative for nonlinear differential operators, Nonlinear Anal. 10 (1986), no. 10, 1083-1103. MR 88f:34024. Zbl 623.34031.

[7] F. Browder, Fixed point theory and nonlinear problems, Proceeding of Symposia in Pure Mathematics, vol. 39, 1983.

[8] N. Tsouli, Etude de l'ensemble nodal des fonctions propres et de la non-résonance pour l'opérateurs p-Laplacien., Ph.D. thesis, Université Mohammed I. Faculté des Sciences. Département de Maths. Oujda. Maroc., 1995.

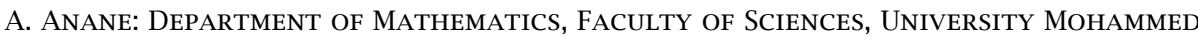
I, OUJDA, MOROCCO

E-mail address: anane@sciences.univ-oujda.ac.ma

N. TSOUli: DePARTMENT OF MATHEMATICS, FACUlTy OF SCIENCES, UNIVERSiTy MOHAMMED

I, OUJDA, MOROCCO

E-mail address: tsou1i@sciences.univ-oujda.ac.ma 


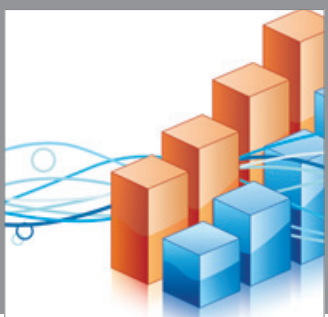

Advances in

Operations Research

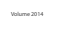

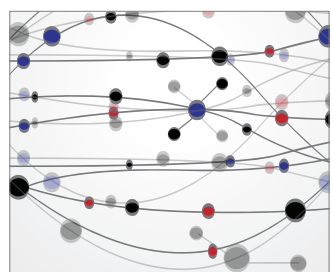

\section{The Scientific} World Journal
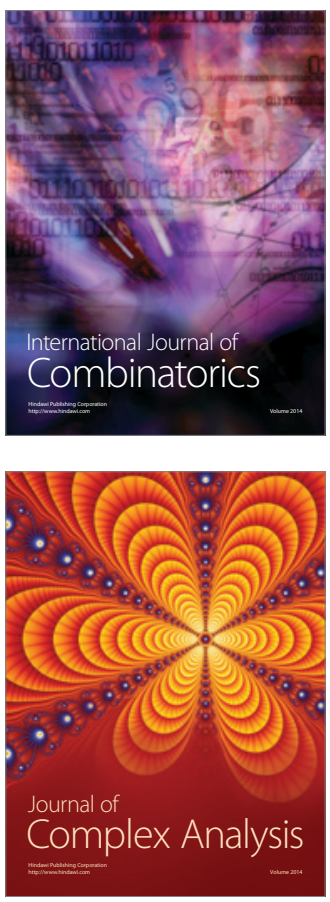

International Journal of

Mathematics and

Mathematical

Sciences
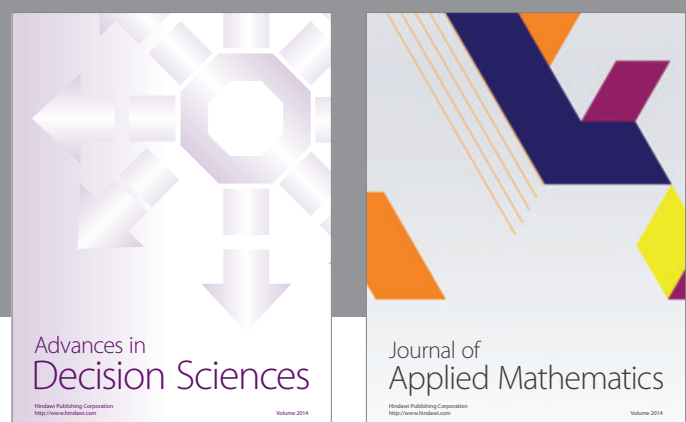

Journal of

Applied Mathematics
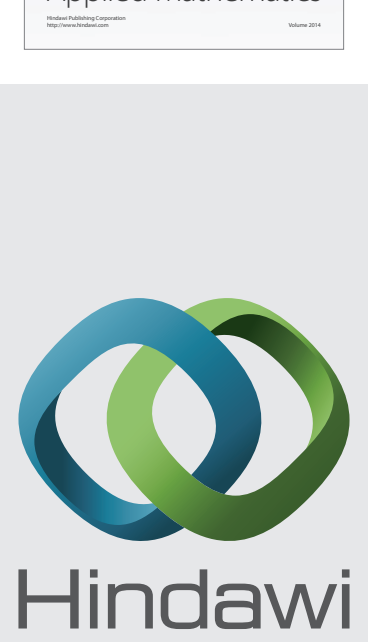

Submit your manuscripts at http://www.hindawi.com
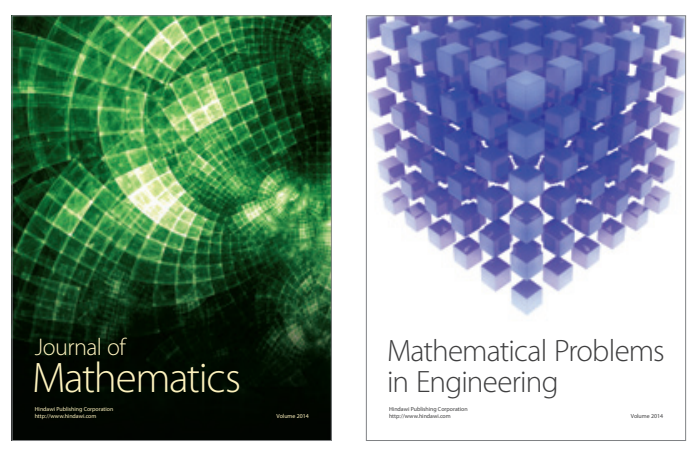

Mathematical Problems in Engineering
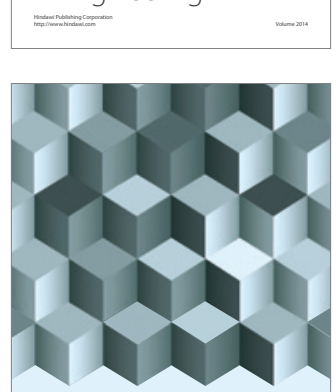

Journal of

Function Spaces
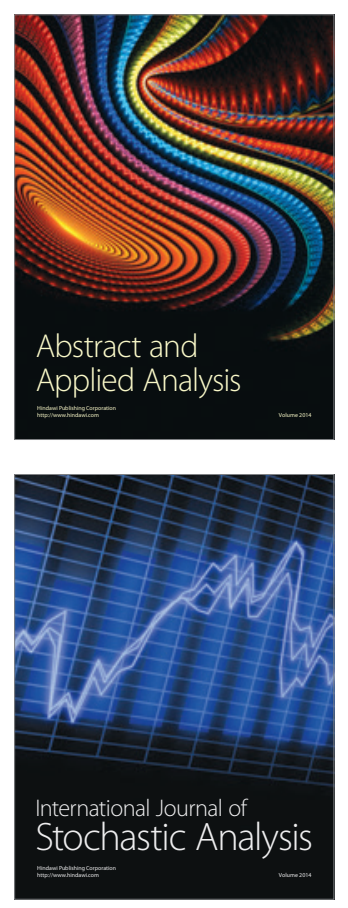

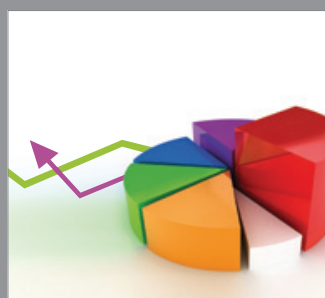

ournal of

Probability and Statistics

Promensencen
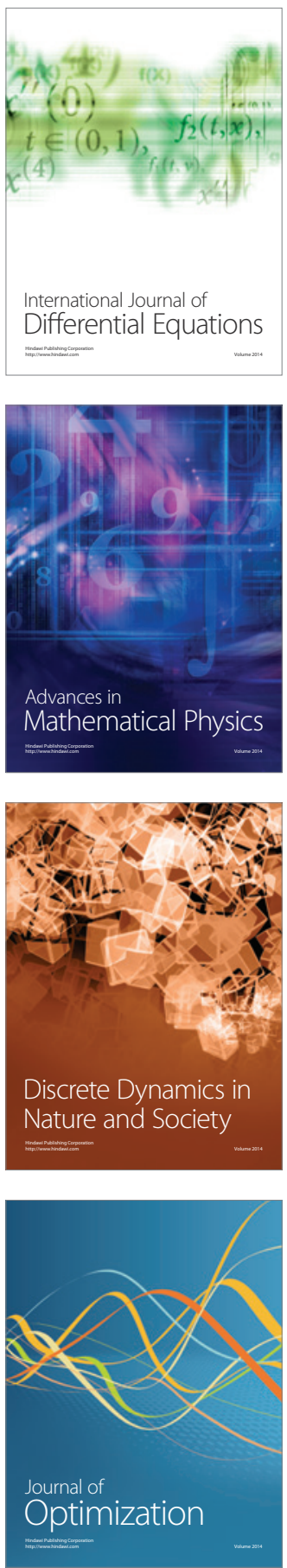